\title{
Inchiesta sulla dialisi peritoneale in Italia
}

\author{
Giuliano Colasanti, Maria Teresa Porri
}

\author{
Divisione di Nefrologia e Dialisi \\ Azienda Ospedaliera San Carlo Borromeo, Milano
}

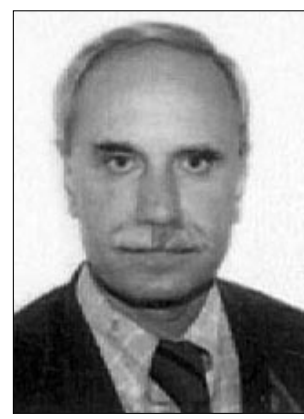

\section{Nel 1998 in Italia solo il $10 \%$ dei pa- zienti ha fruito della dialisi peritoneale (DP) come tec- nica sostituti- va. Perché?}

A nostro avviso in Italia ci sono grandi differenze fra i vari Centri: alcune équipes fortemente motivate hanno iniziato la dialisi peritoneale fin dai primi anni '80 e successivamente hanno seguito i suoi sviluppi e perfezionamenti estendendo la metodica a un numero sempre maggiore di pazienti; altre équipes non ci hanno creduto fin dall'inizio e non l'hanno mai iniziata o peggio, hanno iniziato senza grande convinzione, abbandonandola ai primi ostacoli.

La creazione di un team per la dialisi peritoneale (nefrologo, infermiere, psicologo, assistente sociale) richiede un grande impegno iniziale sia organizzativo che logistico ma soprattutto con motivazioni tenute sempre vive e quindi durature nel tempo.

È necessario che all'interno del team vi sia una profonda conoscenza oltre che del malato, anche del lavoro di ciascuno dei componenti, con affiatamento operativo che poi il paziente osserva come messaggio positivo.

Perché questa percentuale praticamente non è variata negli ultimi quindici anni, nonostante il cambiamento del contesto sociosanitario ed economico?

Perché a mio avviso hanno continuato a fare dialisi peritoneale quei Centri che già la facevano inizialmente, eventualmente solo aumentando il numero dei pazienti.

Nel nostro Centro non c'è stata una flessione positiva nel tempo dell'APD: in primo luogo perché il paziente ideale (giovane, o che lavora) è sempre più introvabile. In secondo luogo perché l'efficienza dialitica dell'APD non è stata per noi certo migliore.

Viene spontaneo pensare che nel nostro Paese la DP sia ancora "a second class treatment, for second class patients by second class doctors"??!

Noi non la consideriamo una metodica di seconda classe ma è sempre più difficile trovare pazienti motivati all'autogestione della propria malattia.

Sembra sempre più diffuso un atteggiamento abbandonico del pa- ziente (vuoi perché più anziano, vuoi perché la struttura familiare di supporto è sempre più assente) che esige la totale gestione della propria terapia da parte della struttura sanitaria.

Non più del $50 \%$ dei pazienti candidati al trattamento sostitutivo, presenta effettive controindicazioni alla DP. Perché nel 1998, in Italia, solo il $18 \%$ dei pazienti incidenti è stato avviato a tale trattamento?

La controindicazione effettiva alla DP nella popolazione che giunge alla nostra osservazione è decisamente superiore al 50\%:

- situazione anatomica addominale controindicante;

- pazienti non idonei alla dialisi domiciliare per problemi clinici e/o sociali.

È importante istituire un ambulatorio di pre-dialisi per individuare i pazienti idonei alla DP mediante colloqui con i medici, gli infermieri e lo psicologo.

Il paziente deve essere informato, rassicurato e incoraggiato a prendersi cura di sé e della propria malattia.

Cattiva organizzazione sanitaria? ... incapacità ad aumentare gli early referred patients, ad at- 
tuare l'incremental dialysis o un integrative care approach?

Impasse politico-amministrativa? "...When either physician or facility reimbursement differences were substantial for one modality, be it haemodialysis or peritoneal dialysis, this was the one utilized to the greatest extent ..."

Problemi di mero interesse economico?

L'impasse politico-amministrativa non ha giocato alcun ruolo nel nostro Centro per la scelta di una dialisi piuttosto che l'altra. Dai responsabili politici e amministrativi viene positivamente recepito il fatto che un paziente in dialisi peritoneale costa meno alla comunità; inoltre nell' ambito del progetto di umanizzazione dell'Ospedale ben si presta la dialisi peritoneale alla possibilità di interventi e miglioramenti.

colasant@tin.it 